\title{
Enfoque espontáneo en estructuras matemáticas: patrones y clasificación
}

\author{
Spontaneous focus on mathematical structures: patterns \\ and sorting
}

Gina Bojorque ${ }^{1}$, Neli Gonzales ${ }^{2}$, Nore Wijns ${ }^{3}$, Lieven Verschaffel ${ }^{4}$, Joke Torbeyns ${ }^{5}$

INFORMACIÓN DEL

\section{ARTÍCULO}

Fecha de recepción: 17 de Agosto de 2021.

Fecha de aceptación: 11 de Noviembre de 2021

1 Doctor of Educational Sciences, KU Leuven. Docente-investigador, Universidad de Cuenca-Ecuador.

E-mail: gina.bojorque@ucuenca.edu.ec Código ORCID:

https://orcid.org/0000-0002-5223-2829

${ }^{2}$ Magister en Gerencia de Proyectos Educativos y Sociales, Universidad de Cuenca. Docente-investigador, Universidad de Cuenca-Ecuador.

E-mail: neli.gonzales@ucuenca.edu.ec Código ORCID

https://orcid.org/0000-0003-3443-8468

3 Doctor of Educational Sciences, y docente-investigador, KU Leuven-Bélgica E-mail: nore.wijns@kuleuven.be Código ORCID:

https://orcid.org/0000-0002-8322-7037

${ }^{4}$ Doctor of Educational Sciences, y docente-investigador, KU Leuven-Bélgica. E-mail: lieven.verschaffel@kuleuven.be Código ORCID:

https://orcid.org/0000-0002-5283-6470

5 Doctor of Educational Sciences, y docente-investigador, KU Leuven-Bélgica. E-mail: joke.torbeyns@kuleuven.be Código ORCID:

https://orcid.org/0000-0003-3244-0265

CITACIÓN: Bojorque, G., Gonzales, N., Wijns, N., Verschaffel, L., \& Torbeyns, J. (2021). Enfoque espontáneo en estructuras matemáticas: patrones y clasificación. Podium, 40, 125-142. doi:10.31095/podium.2021.40.8

ENLACE DOI:

http://dx.doi.org/10.31095/podium.202 1.40 .8

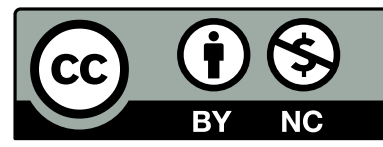

\section{Resumen}

El propósito del estudio fue examinar si los niños preescolares se enfocan espontáneamente en estructuras matemáticas -patrones y clasificación- y si dicho enfoque se asocia con sus habilidades para trabajar patrones. La metodología fue cuantitativa de tipo descriptivo y comparativo. Participaron en el estudio 60 preescolares que fueron evaluados al finalizar el año escolar. Los resultados indicaron que la mayoría de ellos se enfoca espontáneamente en crear una estructura matemática y que no existe asociación entre su tendencia a enfocarse en estructuras matemáticas y su habilidad para trabajar patrones. No se observaron diferencias en el enfoque espontáneo relacionadas al género ni al nivel socioeconómico. Se concluye que estos resultados permiten apuntar a la naturaleza universal del enfoque espontáneo en estructuras matemáticas.

\section{Palabras Clave:}

Estructuras matemáticas, patrones, clasificación, preescolar, niños, nivel socioeconómico.

\section{Clasificación JEL: I0, I2.}

\section{Abstract}

The study aimed to examine whether preschool children spontaneously focus on mathematical structures - patterns and classification - and whether that spontaneous focus is associated with their patterning abilities. The methodology was quantitative, descriptive, and comparative. Participants included 60 preschoolers who were evaluated at the end of the school year. The results indicated that most of them spontaneously focus on creating a mathematical structure and that there is no association between their tendency to focus on mathematical structures and their patterning abilities. No differences were observed in the spontaneous focus related to gender or socioeconomic background. It is concluded that these results allow us to point to the universal nature of the spontaneous focus on mathematical structures.

\section{Keywords:}

Mathematical structures, patterns, sorting, preschool, children, socioeconomic background.

JEL Classification: I0, I2.

$$
125
$$




\section{Introducción}

Desde temprana edad los niños han demostrado ser capaces de desarrollar competencias matemáticas amplias $\mathrm{y}$ sofisticadas, por ejemplo, identificar inmediatamente el número de puntos en un dado o dividir un grupo de galletas en partes iguales (Clements \& Sarama, 2014). En relación a las competencias matemáticas los autores han distinguido dos procesos centrales: las habilidades matemáticas y las disposiciones matemáticas (Bojorque, Torbeyns, Hannula-Sormunen, Van Nijlen, y Verschaffel, 2017; Mulligan et al., 2018; Verschaffel, Rathe, Wijns, De Smedt, y Torbeyns, 2018). Las habilidades matemáticas hacen referencia a los conocimientos y destrezas de los niños, por ejemplo, la habilidad para contar, comparar o sumar. Por otro lado, las disposiciones matemáticas se refieren a la inclinación a enfocarse en elementos matemáticos del entorno, por ejemplo, la inclinación espontánea de mirar números en situaciones cotidianas. Si bien los estudios sobre las competencias matemáticas se han centrado en su mayoría en describir y analizar las habilidades matemáticas de los niños (ej., Andrews \& Sayers, 2015; Aunio y Niemivirta, 2010; Geary, 2011), varios estudios recientes se han enfocado en estudiar el factor de disposición hacia las matemáticas (ej., Bojorque et al., 2017; Degrande, Verschaffel y Van Dooren, 2017; Hannula-Sormunen, 2015; Rathé, Torbeyns, De Smedt, y Verschaffel, 2019). Dentro del aspecto disposicional, la mayoría de estudios se han centrado en investigar el enfoque espontáneo en la numerosidad (véase, por ejemplo, Bojorque et al., 2017; HannulaSormunen, 2015), aunque estudios recientes han abordado la tendencia de los niños a enfocarse espontáneamente en símbolos numéricos (Rathé et al., 2019), en relaciones numéricas (Degrande et al., 2017) o en patrones y estructuras matemáticas (Sharir, Mashal, y Mevarech, 2015; Wijns, De Smedt, Verschaffel, y Torbeyns 2019a).

Con respecto al enfoque espontáneo en estructuras matemáticas, éste ha demostrado estar correlacionado con las habilidades de los niños para trabajar patrones, así como, con sus habilidades matemáticas posteriores (Wijns et al., 2019a; Sharir et al, 2015). A pesar de su importancia, el enfoque espontáneo en estructuras matemáticas ha recibido limitada atención por parte de los investigadores. Hasta donde se sabe, solamente dos estudios el de Sharir et al. (2015) y el de Wijns et al. (2019a), han intentado medir explícitamente el enfoque espontáneo en estructuras matemáticas de los niños preescolares. Más aún, estos estudios se han llevado a cabo en países desarrollados, por lo que no se cuenta con información sobre este tema en países en vías de desarrollo. El propósito del presente estudio fue abordar estas limitaciones al examinar el enfoque espontáneo en estructuras matemáticas de los niños ecuatorianos, con énfasis en patrones y clasificación, y analizar si dicho enfoque guarda relación con las habilidades de los niños en una prueba de patrones matemáticos. 


\section{Revisión de literatura}

\section{Estructuras matemáticas}

En general, el desarrollo de las competencias matemáticas depende en gran medida del pensamiento estructural del niño, el cual permite reconocer, organizar, usar, explicar y conectar elementos o propiedades de una relación matemática (Mason, Stephens y Watson, 2009; Papic, Mulligan, y Mitchelmore, 2011; Mulligan, Papic, Prescott, y Mitchelmore, 2006). Por ejemplo, los niños necesitan reconocer la estructura matemática subyacente para comprender cómo se organiza el sistema numérico al agrupar decenas, o cómo la organización de grupos iguales forma la base de los conceptos de multiplicación y división (Mulligan y Mitchelmore, 1997). De acuerdo a Mulligan, Prescott, y Mitchelmore (2004), la estructura matemática se puede observar en distintas áreas de la matemática como en el número (ej., conteo estructurado en múltiplos: 2 - 4 - 6 - 8), en el espacio (ej., un arreglo triangular de seis puntos), o en la medida (ej., completar una regla para medir). Mulligan y Mitchelmore (2009) concluyeron que los niños que han desarrollado una conciencia de la estructura en un área del aprendizaje de las matemáticas también tienden a mostrar una conciencia estructural en otras áreas.

Varias investigaciones a nivel internacional han estudiado el desarrollo de las estructuras matemáticas tempranas en niños pequeños, las cuales incluyen entre otras, a los patrones (ej., Clements \& Sarama, 2009; Lüken, 2012; Nguyen et al., 2016; Papic, 2007; Rittle-Johnson, Fyfe, Hofer y Farran, 2017; Wijns, Torbeyns, Bakker, De Smedt, y Verschaffel, 2019b). Estas investigaciones han demostrado que los patrones desarrollados a temprana edad son predictores importantes del rendimiento matemático posterior por lo que resulta imprescindible trabajar dichos patrones a temprana edad. Aunque existen diferentes tipos de patrones matemáticos como patrones de repetición (ej., rojo, azul, rojo, azul), patrones de crecimiento (ej., 3, 6, 9, 12) y patrones de estructura espacial, los patrones de repetición son los que más se han trabajado e investigado en el preescolar debido a su facilidad y accesibilidad para niños pequeños (Wijns, Torbeyns, De Smedt, y Verschaffel, 2019c). Entre los tipos de tareas empleadas para trabajar o evaluar los patrones de repetición se encuentran: crear, copiar (o duplicar), interpolar, extender (o continuar), generalizar (o transferir), e identificar la unidad mínima del patrón (Lüken, 2018; Papic et al., 2011; Rittle-Johnson, Fyfe, McLean, y McEldoon, 2013; Wijns et al., 2019b).

Estudios previos que ha examinado el desempeño de los niños en tareas de patrones de repetición han evidenciado que una tendencia usual de los niños en estas tareas es clasificar el material entregado (como fichas, bloques o cubos) atendiendo su color o a su forma (Collins \& Laski, 2015; Rittle-Johnson, Fyfe, Loehr \& Miller, 2015; Rittle-Johnson et al., 2013). Por ejemplo, Collins y Laski (2015) encontraron que, durante las tareas de extender, transferir o identificar la unidad del patrón, el $20 \%$ de los niños 
entre las edades de 3 a 7 años no lograron crear los patrones solicitados y, en su lugar, realizaron otras actividades como clasificar los bloques y cubos, ya sea por la forma o por el color. De igual manera, Rittle-Johnson et al. (2013; 2015) observaron que una acción común de los niños preescolares de 4 a 5 años, durante la realización de tareas de copiar, extender, generalizar o identificar la unidad del patrón, fue clasificar las fichas por su color o forma (los autores puntuaron este tipo de acción como un error en las tareas solicitadas). La clasificación es una habilidad procesal que emplean los niños para ayudarse a organizar el mundo que les rodea, en dicho proceso los niños determinan cómo los objetos pueden ser agrupados en base a un criterio (Platz, 2004). Tanto en los patrones como en la clasificación existe una estructura matemática subyacente, la cual hace referencia a la forma en que se organizan y relacionan los distintos elementos (Mulligan y Mitchelmore, 2009; Mulligan, Mitchelmore, English, y Robertson, 2010).

\section{Enfoque espontáneo en estructuras matemáticas}

Como se indicó anteriormente, el enfoque espontáneo en aspectos matemáticos, ha tomado fuerza por los trabajos de Hannula y colaboradores (Hannula y Lehtinen, 2005; HannulaSormunen, Lehtinen, y Räsänen, 2015), quienes se centraron en investigar el aspecto numérico de las matemáticas. Hannula (2005) definió al enfoque espontáneo como la capacidad de los niños para enfocarse espontáneamente en las características matemáticas (numéricas) de un conjunto de elementos del entorno, aunque no se los guíe explícitamente para hacerlo. A partir del trabajo de Hannula y colaboradores se han llevado a cabo otras investigaciones centradas en estudiar el enfoque espontáneo de los niños en otros aspectos matemáticos, entre ellos, en enfoque espontáneo en estructuras matemáticas (Sharir et al., 2015) y en patrones (Wijns et al., 2019a).

En el estudio de Sharir et al. (2015), las autoras analizaron el reconocimiento espontáneo de estructuras matemáticas de niños de 4 a 6 años, para ello, examinaron tres aspectos de la estructura matemática, a decir, reconocimiento de cantidades (ej., describir una foto con cuatro leones), patrones (ej., describir una foto con tres grupos de dos camellos) y series aritméticas (ej., describir una foto con una serie conformada por un peluche, dos peluches y tres peluches sentados en un mismo sofá). Cada uno de estos aspectos se evaluó mediante tres tipos de tareas, dos tareas verbales y una no verbal. En el primer grupo de tareas verbales se les pidió a los niños describir lo observado en fotos de objetos o animales en su entorno natural. En el segundo grupo de tareas verbales se solicitó a los niños describir figuras geométricas (cuadrados) presentados en tarjetas. Finalmente, en las tareas no verbales el examinador insertó discos de colores en una alcancía y se les pidió a los niños hacer exactamente lo que hizo el examinador. Los resultados señalaron que cerca de la mitad de los niños pudieron reconocer espontáneamente cantidades, un porcentaje 
menor de niños, es decir un cuarto de ellos, reconoció patrones de forma espontánea y un quinto de ellos reconoció espontáneamente series aritméticas. Además, el desempeño de los niños en las tareas de enfoque espontáneo en estructuras predijo su desempeño en una prueba de razonamiento matemático. De acuerdo a Wijns et al. (2019a), las tareas desarrolladas por Sharir y colaboradores no cumplen con los criterios para ser tareas que abordan tendencias de enfoque matemático espontáneo, ya que las tareas no verbales presentaron escasos elementos no matemáticos en los que enfocarse mientras que la tarea verbal dependía en gran medida de la memoria de trabajo de los niños. Según Hannula-Sormunen (2015) las tareas que evalúen el enfoque espontáneo en aspectos matemáticos (numéricos) deben ser novedosas y no explícitamente matemáticas y no deben exceder la capacidad de memoria de trabajo de los niños.

En la investigación de Wijns et al. (2019a), los autores evaluaron el enfoque espontáneo en patrones de niños preescolares de 4 años de edad y su relación con habilidades para trabajar patrones y con habilidades matemáticas. Para evaluar en enfoque espontáneo en patrones los autores emplearon la tarea de la Torre que consistía en colocar delante del niño 15 cubos conectables de tres colores diferentes y pedirle que arme una torre empleando todos lo cubos. Esta tarea aborda las críticas realizadas al trabajo de Sharir et al. (2015) ya que brinda la posibilidad de enfocarse en diferentes elementos matemáticos y no requiere de una fuerte carga de memoria de trabajo. Además, administraron una prueba de patrones y otra de matemáticas. Los hallazgos del estudio indicaron que el $38 \%$ de los preescolares se enfocaron espontáneamente en crear patrones y que existía una asociación significativa entre el enfoque espontáneo en patrones y su desempeño tanto en la prueba de patrones como en la de habilidades matemáticas. A más de los estudios reportados, otros estudios observacionales reportaron que los niños se enfocan espontáneamente en crear patrones en situaciones de juego (Fox, 2005; Seo y Ginsburg, 2004).

En el estudio de Wijns et al. (2019a), los autores observaron que, durante las tareas de enfoque espontáneo en patrones, el $14 \%$ de los niños se enfocaron en clasificar espontáneamente los cubos conectables en base a su color. Adicionalmente, reportaron que aquellos niños que realizaron un arreglo clasificatorio se desempeñaron mejor en la prueba de habilidades para trabajar patrones que los niños que hicieron un arreglo aleatorio. Aparte del estudio de Wijns y colaboradores, otros estudios observacionales también encontraron indicios de enfoques espontáneos de clasificación en niños preescolares. Por ejemplo, Seo y Ginsburg (2004) encontraron que, los niños entre 4 y 5 años de edad tendían a realizar espontáneamente actividades de clasificación durante el juego libre. Además, se ha encontrado que los niños frecuentemente clasifican objetos (en lugar de hacer un patrón) como respuesta a las actividades de creación de patrones (por ejemplo, Collins y Laski, 2015; Rittle-Johnson et al., 2013; 2015). 


\section{El presente estudio}

Como se indicó anteriormente, estudios previos han reportado que el enfoque espontáneo de los niños en estructuras matemáticas se relaciona positivamente con sus habilidades para trabajar patrones, así como, con sus habilidades matemáticas futuras (Wijns et al., 2019a); Sharir et al, 2015). Sin embargo, el enfoque espontáneo en estructuras matemáticas ha sido escasamente estudiado pues se han reportado solamente dos estudios sobre este tema, llevados a cabo en países desarrollados, el de Sharir et al. (2015) y el de Wijns et al. (2019a). En el contexto latinoamericano no se ha realizado ningún estudio al respecto. Por lo expuesto, el propósito del presente estudio fue contribuir al escaso conocimiento existente sobre el enfoque espontáneo en patrones matemáticos de los niños pequeños al examinar si los niños ecuatorianos se enfocan espontáneamente en estructuras matemáticas (patrones y clasificación) y si existe una asociación entre dicha tendencia a enfocarse en estructuras y sus habilidades para trabajar patrones.

Por otro lado, estudios previos sobre el enfoque espontáneo en aspectos matemáticos han reportado diferencias individuales amplias de los niños pequeños en dicho enfoque (Bojorque et al., 2017; Hannula, Lepola, \& Lehtinen, 2010; Wijns et al. 2019a). De acuerdo a Hannula (2005) para algunos niños el mundo puede parecer estar lleno de oportunidades para practicar habilidades matemáticas tempranas mientras que otros pueden enfocarse en otras características del entorno e involucrarse mucho menos con ideas pre-matemáticas. Esta heterogeneidad en edades tempranas es motivo de gran preocupación, dado que está bien documentado la relación entre el enfoque espontáneo de los niños en aspectos matemáticos y sus habilidades matemáticas (Bojorque et al., 2017; Hannula et al., 2010, 2015; Wijns et al. 2019a). Por ello, en este estudio también examinamos si existen diferencias individuales entre los niños ecuatorianos al enfocarse espontáneamente en estructuras matemáticas.

Por último, investigaciones anteriores han examinado si determinadas variables personales y contextuales de los niños, como el género $y$ el nivel socioeconómico familiar, influyen en su enfoque espontáneo en aspectos matemáticos (ej., Seo y Ginsburg, 2004; Wijns et al., 2019a). Seo y Ginsburg (2004) encontraron que ni el género ni el nivel socioeconómico se relacionaron con la frecuencia en la que los niños se enfocaban voluntariamente en realizar actividades matemáticas, incluyendo patrones y clasificación. Por su parte, Wijns et al. (2019a) encontraron diferencias de género en el enfoque espontáneo en patrones de niños, siendo las niñas las que se enfocaron en mayor medida en crear patrones, pero no encontraron diferencias de género en el enfoque espontáneo en la clasificación. Los autores no reportaron diferencias relacionadas al nivel socioeconómico en el enfoque espontáneo en patrones ni en clasificación. En este estudio analizamos si existen diferencias de género y de nivel 
socioeconómico en el enfoque espontáneo en estructuras matemáticas de los niños.

En base a los objetivos propuestos se han planteado cuatro preguntas de investigación:

(1) ¿En qué medida los niños se enfocan espontáneamente en crear estructuras matemáticas: patrones y clasificación?

(2) ¿Existen diferencias individuales en la tendencia a enfocarse en estructuras matemáticas de los niños?

(3) ¿Existe asociación entre la tendencia a enfocarse en estructuras matemáticas de los niños y su habilidad para trabajar patrones?

(4) ¿Existen diferencias de género y de nivel socioeconómico en el enfoque espontáneo en estructuras matemáticas de los niños?

\section{Metodología}

El enfoque de investigación del presente estudio es cuantitativo de tipo descriptivo y comparativo. Los participantes fueron 60 niños (32 varones; 28 mujeres) de preescolar, provenientes de seis instituciones educativas (dos públicas urbanas, dos públicas rurales y dos privadas) de Cuenca-Ecuador. La edad media de los niños al momento de la recolección de datos fue de 5 años, 1 mes ( $\mathrm{DE}=3,2$ meses). En cada institución educativa, se seleccionaron al azar 10 niños. Previo al inicio del estudio, se recopilaron los consentimientos informados de los representantes de cada niño participante.
El nivel socioeconómico de los participantes se construyó en base al nivel de instrucción de la madre (Aunio y Niemivirta, 2010; Bojorque, Torbeyns, Van Hoof, Van Nijlen y Verschaffel, 2019; Starkey, Klein y Wakeley, 2004); para ello se establecieron tres categorías: 1) educación básica (35\% de las madres culminaron este nivel); 2) bachillerato $(26.7 \%$ de las madres culminaron este nivel); y, 3) superior $(38.3 \%$ de las madres culminaron este nivel).

\section{Instrumentos}

\section{Tareas de enfoque espontáneo en patrones}

Para evaluar el enfoque espontáneo en estructuras matemáticas se emplearon dos tipos de tareas: La tarea de la Torre y la tarea de la Oruga (Wijns et al., 2019a). Estas tareas tienen como objetivo capturar la atención espontánea de los niños en los patrones en situaciones basadas en el juego. Por lo tanto, es importante no mencionar la palabra "patrón" cuando se administran las mismas.

La tarea de la Torre consiste en presentar a los niños 15 cubos conectables sueltos de tres colores diferentes (cinco rojos, cinco azules y cinco amarillos). Todos los cubos deben estar colocados en la mesa, frente al niño. El evaluador debe asegurarse de que los cubos no estén clasificados por color, ni unidos entre sí. La consigna dada al niño es sencilla: "Por favor, construye una torre que vaya hacia arriba, usando todos los cubos". Una vez que el niño termina de construir la torre, se le dice que construyó una bonita torre y se toma una fotografía de la misma. 
La tarea de la Oruga consta de una oruga de madera con 15 hoyos en su cuerpo y 15 fichas en 3 colores diferentes (cinco rojas, cinco verdes y cinco azules). El examinador coloca la oruga y todas las fichas en la mesa, frente al niño, asegurándose de que las fichas no estén clasificadas por color. La consigna es la siguiente: "Esta oruga perdió sus colores, ¿puedes por favor devolverle sus colores? Puedes usar todos estos colores". Una vez que el niño coloca todas las fichas en el cuerpo de la oruga, se le dice que la oruga quedó muy contenta y se toma una fotografía de la misma.

Las creaciones de los niños en estas tareas se clasificaron en dos categorías: enfoque en la estructura o creación aleatoria. Se categorizó como "enfoque en la estructura" cuando los niños: (a) crearon un "patrón" es decir, una secuencia de al menos dos unidades completas de un patrón y el comienzo de una tercera unidad (por ejemplo, ABABA o ABBABBA). La secuencia de dos unidades y el inicio de una tercera podrían estar en cualquier lugar de la torre o de la oruga; y (b) "clasificación" es decir, agruparon los cubos o fichas por color (por ejemplo, cinco cubos/fichas de color rojo, cinco amarillos y cinco azules). Se categorizó como "aleatorio" cuando los cubos de las torres o las fichas de las orugas no tenían un patrón (como se indicó en el punto a) o no estaban clasificados (como se indicó en el punto b; ver Tabla 1 para definición y ejemplos

Tabla 1.

Categorización de las respuestas de los niños en las tareas de la Torre y la Oruga

\begin{tabular}{|c|c|c|c|c|}
\hline & Categoría & Descripción & $\begin{array}{c}\text { Ejemplos } \\
\text { Torre }\end{array}$ & $\begin{array}{c}\text { Ejemplos } \\
\text { Oruga } \\
\end{array}$ \\
\hline & Patrón & $\begin{array}{l}\text { Completa una secuencia de al } \\
\text { menos dos unidades } \\
\text { completas de un patrón y el } \\
\text { comienzo de una tercera } \\
\text { unidad. }\end{array}$ & & \\
\hline 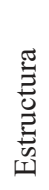 & Clasificación & $\begin{array}{l}\text { Agrupa los cubos o fichas por } \\
\text { color. Por ejemplo, cinco } \\
\text { rojos cinco amarillos y cinco } \\
\text { azules. }\end{array}$ & & \\
\hline 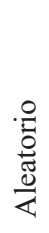 & & $\begin{array}{l}\text { Coloca los cubos o fichas al } \\
\text { azar, sin un patrón o } \\
\text { clasificación. }\end{array}$ & $\theta$ & \\
\hline
\end{tabular}

Fuente: Elaboración propia. 
de estas categorías). Para evaluar la confiabilidad en la calificación de las tareas, dos evaluadoras independientes puntuaron el $17 \%$ de las Torres realizadas por los niños y el 17\% de las Orugas, y estuvieron de acuerdo en todas, lo que indica una fiabilidad perfecta entre evaluadoras $(\mathrm{K}=1,00)$. A continuación, una evaluadora calificó todas las producciones de los niños en la tarea de la Torre y otra evaluadora todas las producciones en la tarea de la Oruga.

La habilidad de los niños para trabajar patrones se evaluó mediante la Prueba de patrones matemáticos de Wijns et al. (2019b), la cual consta de tres tipos de tareas. La primera, extender patrones, implica escoger entre cuatro opciones presentadas, la figura que continúa correctamente un determinado patrón. La segunda, generalizar, involucra copiar un patrón modelo empleando tarjetas con figuras diferentes a las de ese patrón. Y, la tercera tarea, reconstruir un patrón (o identificar la unidad del patrón) implica observar un patrón modelo durante pocos segundos, recordarlo y reproducirlo. Cada una de estas tres tareas consta de seis ítems (es decir, $\mathrm{AB}, \mathrm{AAB}, \mathrm{ABC}$, $\mathrm{AABB}, \mathrm{AABC}$ y $\mathrm{ABCD}$ ) dando lugar a 18 ítems (ver Tabla 2 para instrucciones y ejemplos de cada tarea). Cada ítem se califica dicotómicamente, es decir, se asigna 1 punto por cada respuesta correcta y 0 puntos por cada respuesta incorrecta. El puntaje máximo a obtener es de 18 puntos. La consistencia interna de la prueba en nuestra muestra fue aceptable ( $\alpha=.70$; véase Field, 2009). La confiabilidad entre evaluadores (después de calificar el $10 \%$ de las pruebas) fue buena $(\mathrm{K}=.92, \mathrm{p}<.001)$.función

\section{Procedimiento}

Al finalizar el año escolar, los niños fueron evaluados en sus propias instituciones educativas, en un aula tranquila, sin distracciones y $\sin$ estímulos matemáticos a su alrededor. La evaluación de cada niño duró alrededor de 25 minutos y la evaluación completa, a todos los niños, tomó tres semanas. Primero se les administró las tareas de enfoque espontáneo en estructuras matemáticas y después la prueba de patrones matemáticos, esto con el fin de prevenir que los niños se enfoquen en patrones en base a la experiencia de haber dado primero la prueba de patrones. Durante la administración de las tareas de la Torre y la Oruga los evaluadores no utilizaron en ningún momento la palabra "patrón" pues la intención de las tareas es evaluar el enfoque espontáneo, no dirigido, en patrones. Todos los niños recibieron primero la tarea de la Torre y luego la tarea de la Oruga. Al finalizar cada tarea, los evaluadores tomaron una fotografía de la creación de los niños acompañada de la parte superior del formulario de puntuación, con el nombre y código único del niño. En base a esa fotografía se analizaron y puntuaron las creaciones de los niños.

\section{Resultados}

Para determinar en qué medida los niños se enfocan espontáneamente en crear estructuras matemáticas: patrones y clasificación (Pregunta de investigación 1) y si existen diferencias individuales en 
Tabla 2.

Instrucciones y ejemplo de cada tarea de patrones

\begin{tabular}{l} 
Tarea e instrucción \\
\hline Extender \\
"Mira detenidamente esta serie. Se caracteriza por \\
un patrón. Al final de la serie, falta una figura. \\
¿Ves qué figura falta? Una de estas figuras debe \\
colocarse en el lugar vacío. ¿Cuál de estas figuras \\
debe colocarse aquí?"
\end{tabular}

Generalizar

"Mira detenidamente esta serie. Se caracteriza por un patrón. Por favor , haz el mismo patrón, usando tus figuras". (Las figuras son diferentes a las del patrón modelo)

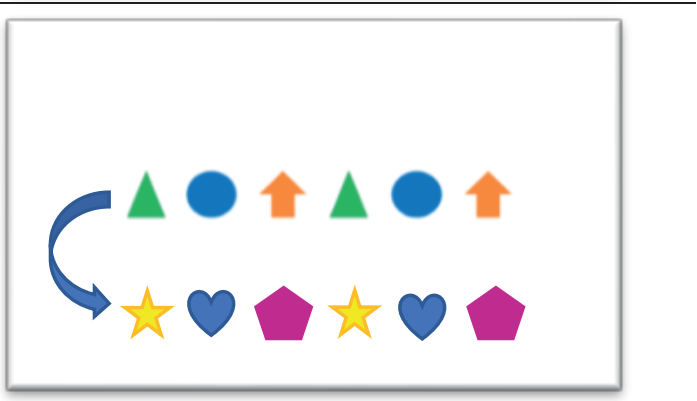

Reconstruir un patrón

"Mira detenidamente esta serie. Se caracteriza por un patrón. La verás solamente por unos segundos. Por favor, recuerda el patrón y reconstruye el mismo patrón con las figuras". (Las figuras iguales a las del patrón modelo)

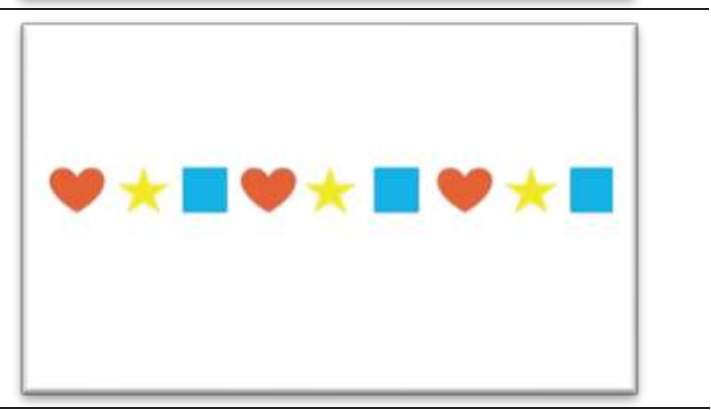

Fuente: Elaboración propia.

la tendencia de los niños a enfocarse en estructuras matemáticas (Pregunta de investigación 2), se procedió a calcular el número y porcentaje de niños que se enfocaron espontáneamente en crear estructuras (patrones o clasificación) o en realizar un arreglo aleatorio en las tareas de enfoque espontáneo en patrones. E1 resultado indica que el $66.7 \%$ de los niños (40 niños) crearon espontáneamente una estructura, es decir, ya sea que crearon un patrón o clasificaron los cubos/fichas por color mientras que, el 33.3\% (20 niños) restante no se enfocó en crear patrones ni en clasificar por color, es decir, realizaron un arreglo aleatorio. De los 40 niños que crearon un patrón o clasificaron los objetos por color, 25 niños lo hicieron sólo en una de las tareas y 14 niños 10 hicieron en las dos tareas. Estos resultados evidencian las diferencias individuales en la tendencia a enfocarse en estructuras y patrones.

A continuación, para examinar si existe asociación entre la tendencia a enfocarse en estructuras matemáticas de los niños y su habilidad para trabajar 
patrones (Pregunta de investigación 3), se procedió, en primer lugar, a determinar la media del puntaje obtenido en la prueba de patrones matemáticos de cada grupo (estructura vs aleatorio). Los resultados indican que, en general, los niños pudieron resolver con precisión alrededor de un tercio de los ítems. El grupo que se enfocó en estructuras obtuvo un puntaje mayor en la prueba de patrones matemáticos $(M=6.83[D E=3.30])$ que el grupo que realizó un arreglo aleatorio $(M=5.90[D E=2.75])$. Sin embargo, la prueba $U$ de Mann-Whitney para determinar la diferencia entre grupos señaló que no hubo diferencias significativas entre los grupos en su habilidad para formar patrones $U=343, p=.37$.

Finalmente, se analizó si los niños diferían en género y nivel socioeconómico en su tendencia a enfocarse en estructuras matemáticas (Pregunta de investigación 4). Los resultados indicaron que no existen diferencias relacionadas al género de los niños $X^{2}=.54, p=.46$; ni al nivel socioeconómico de sus familias, $X^{2}=$ $3.43, p=.33$.

En el presente estudio se examinó si los niños ecuatorianos de preescolar se enfocan espontáneamente en crear estructuras matemáticas - patrones $\mathrm{y}$ clasificación - y si se evidencia una asociación entre dicho enfoque y sus habilidades para trabajar patrones. Además, se analizó si existen diferencias individuales en la tendencia de los niños a enfocarse en estructuras matemáticas, así como diferencias de género y nivel socioeconómico.
Los resultados indican que la mayoría de niños de este estudio se enfocaron espontáneamente en crear estructuras matemáticas, ya sea en forma de patrones o de clasificación. Este resultado está en línea con lo reportado en el estudio de Wijns et al. (2019a) en Flanders-Bélgica, quienes observaron que el $52 \%$ de los niños preescolares se enfocaron espontáneamente en crear ya sea un patrón o un arreglo clasificatorio en la tarea de la Torre (empleada en este estudio) que evalúa el enfoque espontáneo en patrones. Aunque en menor porcentaje, Sharir et al. (2015) también encontraron que los niños israelitas de 4 a 6 años se enfocaron espontáneamente en reconocer estructuras matemáticas en forma de cantidades, patrones y series aritméticas. Los resultados de este estudio también concuerdan con estudios observacionales previos, llevados a cabo en Australia y USA, que encontraron manifestaciones de enfoques espontáneos en patrones $\mathrm{y}$ clasificación en niños preescolares durante actividades de juego libre (Fox, 2015; Seo y Ginsburg, 2004). Los hallazgos del presente estudio contribuyen a la teoría existente sobre el enfoque espontáneo en aspectos matemáticos al demostrar que, al igual que en los países recientemente mencionados, un alto porcentaje de niños ecuatorianos presentaron una tendencia natural a enfocarse espontáneamente en estructuras matemáticas en su entorno y que existen diferencias individuales en dicho enfoque.

Al igual que en otros estudios sobre el enfoque espontáneo en aspectos matemáticos (ej., Bojorque et al., 2017; 
Hannula et al., 2010; Wijns et al. 2019a), en el presente estudio se encontraron diferencias individuales en la tendencia a enfocarse en estructuras matemáticas, lo que indica que, desde temprana edad, los niños difieren en la tendencia a prestar atención a estructuras - como patrones en su entorno. Estas diferencias son preocupantes, ya que estudios previos han demostrado una asociación entre enfoque espontáneo en estructuras y diferentes habilidades matemáticas de los niños (Sharir et al., 2015; Wijns et al., 2019a). Entonces, futuros estudios deberían centrarse en implementar en los centros educativos actividades específicas que faciliten el enfoque espontáneo en estructuras matemáticas con miras a estimular dicha tendencia en todos los niños pequeños. Los estudios de Hannula et al. $(2005 ; 2020)$ y de Bojorque et al. (2018) pueden servir de ejemplo para implementar una estimulación adecuada en este sentido. En los estudios de Hannula et al. (2005; 2020), los autores demostraron que fue posible aumentar la tendencia de los niños pequeños a enfocarse espontáneamente en aspectos numéricos de su entorno, mediante actividades cotidianas $\mathrm{y}$ fáciles de incorporar en la jornada diaria de los centros infantiles. De igual manera, el estudio de Bojorque y colaboradores (Bojorque, Torbeyns, Van Hoof, Van Nijlen \& Verschaffel, 2018) reportó una mejora en el enfoque espontáneo en el número de los niños de jardín infantil después de haber implementado un programa de estimulación de competencias matemáticas que, entre otros aspectos, estimuló el enfoque espontáneo en la numerosidad.
Por otro lado, a diferencia de lo reportado por Wijns et al. (2019a), en el presente estudio no se observó una asociación significativa entre la tendencia a enfocarse en estructuras matemáticas y las habilidades de los niños para trabajar patrones. Este resultado podría deberse a que el enfoque espontáneo, es una tendencia natural, no enseñada. Por el contrario, el desempeño en tareas de patrones requiere poner en juego habilidades para trabajar patrones, las cuales, por lo general, se aprenden de manera explícita en los centros educativos. En este caso, se observó que dos tercios de los niños crearon de manera espontánea patrones o clasificaron, pero solamente pudieron resolver correctamente un tercio de los ítems de la prueba de patrones matemáticos, demostrando un bajo nivel de desempeño en esta prueba, lo cuál podría estar asociado a las reducidas oportunidades para trabajar patrones que brinda el currículo ecuatoriano de preescolar (Bojorque y Gonzales, 2021). Una limitación metodológica del presente estudio fue que la habilidad para trabajar patrones se evaluó mediante una prueba más amplia y, por lo tanto, también más válida y confiable, que la pruebas tareas para evaluar enfoque espontáneo en estructuras matemáticas, la cual incluyó solamente dos actividades.

En este estudio no se evidenciaron diferencias asociadas al género ni al nivel socioeconómico en la tendencia de los niños a enfocarse espontáneamente en estructuras matemáticas, lo que concuerda con lo reportado por Seo y Ginsburg (2004), quienes tampoco 
observaron diferencias de género ni de nivel socioeconómico en la frecuencia en la que los niños se enfocaban voluntariamente en realizar actividades matemáticas como crear patrones $\mathrm{y}$ clasificar. Este resultado también coincide, en parte, con Wijns et al. (2019a) quienes no encontraron diferencias de género en el enfoque espontáneo en patrones, aunque estos autores sí reportaron diferencias de género siendo las niñas las que se enfocaron en mayor medida en crear patrones. Debido a que existen pocos estudios sobre la influencia del nivel socioeconómico y del género en la tendencia a enfocarse en estructuras matemáticas, es necesario realizar futuras investigaciones que permitan determinar con mayor profundidad el rol que juegan estas dos variables. Esas investigaciones deberían incluir información sobre el entorno matemático del hogar con miras a determinar si la estimulación que los niños reciben en sus hogares influye con su inclinación a enfocarse espontáneamente en estructuras matemáticas y si dicha estimulación difiere entre niños y niñas.

\section{Conclusiones}

Una primera conclusión del estudio es que los resultados obtenidos permiten apuntar a la naturaleza universal del enfoque espontáneo en estructuras matemáticas al demostrar que, al igual que en países desarrollados, social, económica y culturalmente diferentes a los países en vías de desarrollo, los niños ecuatorianos presentan una tendencia natural a enfocarse en estructuras matemáticas. Estas similitudes indican que existen mecanismos similares que subyacen al desarrollo del enfoque espontáneo en estructuras matemáticas de los niños en contextos educativos diferentes. Una segunda conclusión es que las grandes diferencias interindividuales de los niños pequeños en su tendencia a enfocarse en estructuras matemáticas presenta una implicación educativa importante que señala la necesidad de que los educadores tomen conciencia del interés espontáneo de los niños en trabajar estructuras matemáticas, con miras a crear situaciones educativas en las cuales todos los niños puedan practicar actividades que refuerce el trabajo con diferentes tipos de estructuras matemáticas como patrones y clasificación, y así recibir una educación en torno a esta área que sea retadora y motivante. Para ello podrían llevarse a cabo estudios de intervención en esta área. Los resultados de tales estudios brindarán la posibilidad de establecer prácticas de educación matemática pertinentes, acordes al contexto ecuatoriano. Otra sugerencia para futuras investigaciones sería llevar a cabo estudios longitudinales que sigan a los niños a lo largo de la escuela primaria para determinar si el enfoque espontáneo en estructuras matemáticas es estable o se modifica con el tiempo.

\section{Fuente de financiamiento de la investigación}

Dirección de Investigación de la Universidad de Cuenca (DIUC).

\section{Contribución de autores}

G.B. Idea, revisión de literatura, 
metodología, análisis de datos, redacción y revisión final del artículo.

N.G. Metodología, análisis de datos, redacción y revisión final del artículo.

N.W. Revisión de literatura, metodología, análisis de datos, redacción y revisión final del artículo.

L.V. Revisión de literatura, metodología, análisis de datos, redacción y revisión final del artículo.

J.T. Revisión de literatura, metodología, análisis de datos, redacción y revisión final del artículo.

\section{Referencias}

Andrews, P., y Sayers, J. (2015). Identifying opportunities for grade one children to acquire foundational number sense: Developing a framework for cross cultural classroom analyses. Early Childhood Education Journal, (43), 257-267. https://doi.org/10.1007/s10643$014-0653-6$

Aunio, P., y Niemivirta, M. (2010). Predicting children's mathematical performance in grade one by early numeracy. Learning and Individual Differences, 20(5), 427-435. https://doi.org/10.1016/j.lindif. 2010.06.003

Bojorque, G., y Gonzales, N. (2021). Patrones matemáticos en los niveles de Inicial y Preparatoria: Análisis del currículo. INNOVA Research Journal, 6(1), 47-60. https://doi.org/10.33890/innova.v6.n1.20 21.1433

Bojorque, G., Torbeyns, J., Hannula-Sormunen, M., Van Nijlen, D., y Verschaffel, L. (2017). Development of SFON in
Ecuadorian Kindergartners. European Journal of Psychology of Education, (32), 449-462. doi: 10.1007/s10212-016-03 06-9

Bojorque, G., Torbeyns, J., Van Hoof, J., Van Nijlen, D., y Verschaffel, L. (2018). Effectiveness of the Building Blocks program for enhancing Ecuadorian kindergartners' numerical competencies. Early Childhood Research Quarterly, 44(3), 231-241. https://doi.org/10.1016/ j.ecresq.2017.12.009

Bojorque, G., Torbeyns, J., Van Hoof, J., Van Nijlen, D., y Verschaffel, L. (2019). Influencia del nivel socioeconómico en el desarrollo de las competencias numéricas de los niños ecuatorianos de jardín infantil. Perfiles Educativos, 41(166), 90 -104. https://doi.org/10.22201/iisue. 2448 6167e.2019.166.59183

Clements, D., y Sarama, J. (2009). Learning and teaching early maths: The learning trajectories approach. NY: Routledge.

Collins, M. A., y Laski, E. V. (2015). Preschoolers' strategies for solving visual pattern tasks. Early Childhood Research Quarterly, 32(3), 204-214. https://doi.org/10.1016/j. ecresq.2015.04.004

Degrande, T., Verschaffel, L., y Van Dooren, W. (2017). Spontaneous focusing on quantitative relations: Towards a characterization. Mathematical Thinking and Learning, 19(4), 260-275. https://d oi.org/10.1080/10986065.2017.1365223

Field, A. (2009). Discovering statistics using SPSS (Third Edition). London, UK: SAGE.

Fox, J. (2005). Child-initiated mathematical patterning in the pre-compulsory years. In H. L. Chick y J. L. Vincent (Eds.), Proceedings of the 29th Conference of the International Group for the Psychology of Mathematics Education (Vol. 2, pp. 313-320). Melbourne, Vic.: PME. 
Geary, D. (2011). Cognitive predictors of achievement growth in mathematics: a 5-year longitudinal study. Developmental Psychology, 47(6), 1539-1552. doi: $10.1037 / \mathrm{a} 0025510$

Hannula, M. M. (2005). Spontaneous focusing on numerosity in the development of early mathematical skills. Annales Universitais Turkuensis B, 282. Painosalama: Turku.

Hannula, M. M., y Lehtinen, E. (2005). Spontaneous focusing on numerosity and mathematical skills of young children. Learning and Instruction, 15(3), 237-256. https://doi.org/10.1016/j.learninstruc. 200 5.04 .005

Hannula, M. M., Lepola, J., y Lehtinen, E. (2010) Spontaneous focusing on numerosity as a domain-specific predictor of arithmetical skills. Journal of Experimental Child Psychology, 107(4), 394-406. DOI: 10.1016/j.jecp.2010.06.004

Hannula, M. M., Mattinen, A., y Lehtinen, E. (2005). Does social interaction influence 3-year-old children's tendency to focus on numerosity? A quasi-experimental study in day-care. In L. Verschaffel, E. De Corte, G. Kanselaar, y M. Valcke (Eds.), Powerful learning environments for promoting deep conceptual and strategic learning (Studia Paedagogica Vol. 41, pp. 63-80). Leuven: Leuven University Press.

Hannula-Sormunen, M. M. (2015). Spontaneous focusing on numerosity and its relation to counting and arithmetic. In R. C. Kadosh \& A. Dowker (Eds.), The Oxford handbook of numerical cognition (pp. 275-290). Oxford, UK: Oxford University Press.

Hannula-Sormunen, M. M., Lehtinen, E., y Räsänen, P. (2015). Preschool children's spontaneous focusing on numerosity, subitizing, and counting skills as predictors of their mathematical performance seven years later at school. Mathematical Thinking and Learning, 17(2-3), 155-177. https://doi.org/10.1080/10986065.2015.1 016814

Hannula-Sormunen, M., Nanu, C., Luomaniemi, K., Heinonen, M., Sorariutta, A., Södervik, I., y Mattinen, A. (2020). Promoting spontaneous focusing on numerosity and cardinality-related skills at day care with one, two, how many and count, how many programs, Mathematical Thinking and Learning, 22(4), 312-331. https://doi.org/10.1080/ 10986065.2020.1818470

Lüken, M. (2012). Young children's structure sense. Journal Für Mathematik-Didaktik, (33), 263-285. https://doi.org/10.1007/ s13138-012-0036-8

Lüken, M. M. (2018). Repeating pattern competencies in three- to five-year old kindergartners: A closer look at strategies. In C. B. Iliada Elia, J. Mulligan, A. Anderson, y A. Baccaglini-Frank (Eds.), Contemporary research and perspectives on early childhood mathematics education (pp. 35-54). Hamburg: Springer.

Mason, J., Stephens, M., y Watson, A. (2009). Appreciating Mathematical structure for all. Mathematics Education Research Journal, 21(2), 10-32.

Mulligan, J. T., y Mitchelmore, M. C. (1997). Young children's intuitive models of multiplication and division. Journal for Research in Mathematics Education, 28(3), 309-330. https://doi.org/10.2307/ 749783

Mulligan, J., Prescott, A., y Mitchelmore, M. (2004). Children's development of structure in early mathematics. In M. J. Høines y A. B. Fuglestad (Eds.), Proceedings of the 28th annual conference of the International Group for the Psychology of Mathematics Education (Vol. 3, pp. 393-400). Bergen, Norway: Program Committee. 
Mulligan, J., y Mitchelmore, M. (2009). Awareness of pattern and structure in early mathematical development. Mathematics Education Research Journal, (21), 33-49. https://doi.org/10.1007/BF03217544

Mulligan, J. T., Mitchelmore, M. C., English, L. D., y Robertson, G. (2010). Implementing a Pattern and Structure Mathematics Awareness Program (PASMAP) in kindergarten. In L. Sparrow, B. Kissane, \& C. Hurst (Eds.), Shaping the future of mathematics education (Proceedings of the 33rd annual conference of the Mathematics Education Research Group of Australasia, pp. 796-803). Fremantle: MERGA.

Mulligan, J. T., Papic, M., Prescott, A., y Mitchelmore, M. C. (2006). Improving early numeracy through a Pattern and Structure Mathematics Awareness Program (PASMAP). In P. Grootenboer, R. Zevenbergen, y M. Chinnappan (Eds.), Identities, cultures and learning spaces (Proceedings of the 29th annual conference of the Mathematics Education Research Group of Australasia, Canberra, Vol. 2, pp. 376-383). Adelaide: MERGA.

Mulligan, J., Verschaffel, L., Baccaglini-Frank, A., Coles, A., Gould, P., He, S., . . . Yang, D. C. (2018). Whole number thinking, learning and development: Neurocognitive, cognitive and developmental approaches. En M. Bussi y X. Sun (Eds.), Primary mathematics study on whole numbers (ICMI Study 23, pp. 137-167). New York, NY: Springer.

Nguyen, T., Watts, T. W., Duncan, G. J., Clements, D., Sarama, J., Wolfe, C., y Spitler, M. E. (2016). Which preschool mathematics competencies are most predictive of fifth grade achievement? Early Childhood Research Quarterly, 36(3), 550-560. http s://doi.org/10.1016/j.ecresq.2016.02.003

Papic, M. (2007). Promoting repeating patterns with young children-more than just alternating colours! Australian Primary Mathematics Classroom, 12(3), 8-13.

Papic, M., Mulligan, J., y Mitchelmore, M. (2011). Assessing the development of preschoolers' mathematical patterning. Journal for Research in Mathematics Education, 42(3), 237-268. https://doi. org/10.5951/jresematheduc.42.3.0237

Platz, D. L. (2004). Challenging young children through simple sorting and classifying: A developmental approach. Education 125(1), 3-13.

Rathé, S., Torbeyns, J., De Smedt, B., y Verschaffel, L. (2019). Spontaneous focusing on Arabic number symbols and its association with early mathematical competencies. Early Childhood Research Quarterly, 48(3), 111-121. https://doi. org/10.1016/j.ecresq.2019.01.011

Rittle-Johnson, B., Fyfe, E. R., Hofer, K. G., y Farran, D. C. (2017). Early math trajectories: Low- income children's mathematics knowledge from age 4 to 11 . Child Development, 88(5), 1727- 1742. https://doi.org/10.1111/cdev.12662

Rittle-Johnson, B., Fyfe, E. R., Loehr, A. M., y Miller, M. R. (2015). Beyond numeracy in preschool: Adding patterns to the equation. Early Childhood Research Quarterly, 31(2), 101-112. https://doi. org/10.1016/j.ecresq.2015.01.005

Rittle-Johnson, B., Fyfe, E. R., McLean, L. E., y McEldoon, K. L. (2013). Emerging understanding of patterning in 4-year-olds. Journal of Cognition and Development, 14(3), 376-396. https:// doi.org/10.1080/15248372.2012.689897

Seo, K.-H., y Ginsburg, H. (2004). What is developmentally appropriate in early childhood mathematics education? Lessons from new research. In D. Clements, J. Sarama, y A.-M. DiBiase (Eds.), Engaging young children in 
mathematics (pp. 91-104). Mahwah, NJ: Lawrence Erlbaum.

Sharir, T., Mashal, N., y Mevarech, Z. R. (2015). To what extent can young children spontaneously recognize mathematical structures? Paper presented at the Bienial Conference of the European Association for Research on Learning and Instruction, Limasol, Cyprus.

Starkey, P., Klein, A., y Wakeley, A. (2004). Enhancing young children's mathematical knowledge through a pre-kindergarten mathematics intervention. Early Childhood Research Quarterly, 19(1), 99-120. https://doi.org/10.1016/ j.ecresq.2004.01.002

Verschaffel, L., Rathe, S., Wijns, N., De Smedt, B., \& Torbeyns, J. (2018). Young children's early mathematical competencies: The role of mathematical focusing tendencies. Invited plenary lecture. Kristiansand, Norway, at the POEM conference.

Wijns, N., De Smedt, B., Verschaffel, L., y Torbeyns, J. (2019a). Are preschoolers who spontaneously create patterns better in mathematics? British Journal of Educational Psychology, 90(3), 753-769. https://doi.org/10.1111/bjep.12329

Wijns, N., Torbeyns, J., Bakker, M., De Smedt, B., y Verschaffel, L. (2019b). Four-year olds' understanding of repeating and growing patterns and its association with early numerical ability. Early Childhood Research Quarterly, 49(4), 152-163. https://doi.org/10.1016/j.ecresq.2019.06. 004

Wijns, N., Torbeyns, J., De Smedt, B., y Verschaffel, L. (2019c). Young children's patterning competencies and mathematical development: A review. In K. Robinson, H. Osana, y D. Kotsopoulos, (Eds.), Mathematical learning and cognition in early childhood (pp. 139- 161). Cham:
Springer International Publishing. https://doi.org/10.1007/978-3-030-12895 $-1 \_9$

PODIUM No. 40, Diciembre 2021, pp. 125-142

(C) Universidad Espíritu Santo - UEES

ISSN: 1390-5473 e-ISSN: 2588-0969 
